Authorship note: Jesús Carcía-Donas, Benoit Beuselinck, and Lucía IngladaPérez contributed equally to this work.

Conflict of interest: J. Carcía-Donas has received honoraria from Pfizer and Novartis and research funding from Pfizer. B. Beuselinck has received honoraria from Pfizer and Novartis and has an advisory role with Novartis. $P$. Schöffski has received institutional research support and institutional honoraria for advisory and educational functions from GlaxoSmithKline (CSK), Novartis, and Bayer. A. Wozniak has received honoraria from Boehringer Ingelheim, and CSK has covered travel and accommodation expenses. $A$. Conzález del Alba has an advisory role with Astellas Pharma, CSK, Sanofi, and Bayer Janssen Oncology, and Astellas Pharma, Pfizer, and Janssen Oncology have covered travel and accommodation expenses. M.A. Climent has an advisory role with Pfizer and Novartis, and Pfizer has covered travel and accommodation expenses. S. Hernando has an advisory role with Pfizer, and Janssen has covered travel and accommodation expenses.

J.A. Arranz has received honoraria from GSK, Pfizer, Astellas Pharma, Pirre Fabre, and Roche Consulting; has an advisory role with CSK, Pfizer, Janssen-Cilag, Astellas Pharma, Sanofi, Novartis, Roche, and Pierre Fabre; and has received research funding from CSK and Sanofi, and Astellas Pharma has covered travel and accommodation expenses. C. Rodriguez-Antona has received research funding from Pfizer.

Submitted: December 18, 2015

Accepted: May 31, 2016

Published: July 7, 2016

Reference information:

JCI Insight. 2016;1(10):e86051.

doi:10.1172/jci.insight.86051.

\section{Deep sequencing reveals microRNAs predictive of antiangiogenic drug response}

\author{
Jesús García-Donas, ,,2 Benoit Beuselinck,, ${ }^{3,4}$ Lucía Inglada-Pérez, ${ }^{5,6}$ Osvaldo Graña, ${ }^{7}$ \\ Patrick Schöffski,3,4 Agnieszka Wozniak, ${ }^{4}$ Oliver Bechter, ${ }^{3,4}$ Maria Apellániz-Ruiz, ${ }^{5}$ \\ Luis Javier Leandro-García, ${ }^{5}$ Emilio Esteban, ${ }^{2,8}$ Daniel E. Castellano, ${ }^{2,9}$
} Aranzazu González del Alba, ,,10 Miguel Angel Climent, ${ }^{2,11}$ Susana Hernando, , ${ }^{2,12}$ José Angel Arranz,, 2,13 Manuel Morente, ${ }^{14}$ David G. Pisano, ${ }^{7}$ Mercedes Robledo, ${ }^{5,6}$ Cristina Rodriguez-Antona ${ }^{5,6}$

'Oncology Unit, HM Hospitales - Centro Integral Oncológico HM Clara Campal, Madrid, Spain. ${ }^{2}$ Spanish Oncology Genitourinary Group, Madrid, Spain. ${ }^{3}$ Department of General Medical Oncology, University Hospitals Leuven, and ${ }^{4}$ Laboratory for Experimental Oncology, KU Leuven, Leuven, Belgium. ${ }^{5}$ Hereditary Endocrine Cancer Group, Human Cancer Genetics Programme, Spanish National Cancer Research Centre, Madrid, Spain. ${ }^{6}$ Centro de Investigación Biomédica en Red de Enfermedades Raras, Madrid, Spain. 'Bioinformatics Unit, Structural Biology and Biocomputing Programme, Spanish National Cancer Research Centre, Madrid, Spain. ${ }^{8} \mathrm{Hospital}$ Universitario Central de Asturias, Oviedo, Spain. ${ }^{9} \mathrm{Hospital}$

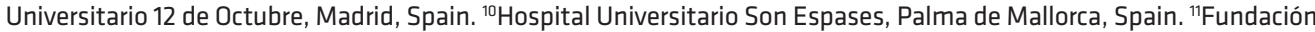
Instituto Valenciano de Oncología, Valencia, Spain. ${ }^{12 H o s p i t a l ~ U n i v e r s i t a r i o ~ F u n d a c i o ́ n ~ A l c o r c o ́ n, ~ M a d r i d, ~ S p a i n . ~}$ ${ }^{13}$ Hospital General Universitario Gregorio Marañón, Madrid, Spain. ${ }^{14}$ Tumour Bank Unit, Spanish National Cancer Research Centre, Madrid, Spain.

The majority of metastatic renal cell carcinoma (RCC) patients are treated with tyrosine kinase inhibitors (TKI) in first-line treatment; however, a fraction are refractory to these antiangiogenic drugs. MicroRNAs (miRNAs) are regulatory molecules proven to be accurate biomarkers in cancer. Here, we identified miRNAs predictive of progressive disease under TKI treatment through deep sequencing of $\mathbf{7 4}$ metastatic clear cell RCC cases uniformly treated with these drugs. Twentynine miRNAs were differentially expressed in the tumors of patients who progressed under TKI therapy ( $P$ values from $6 \times 10^{-9}$ to $\left.3 \times 10^{-3}\right)$. Among 6 miRNAs selected for validation in an independent series, the most relevant associations corresponded to miR-1307-3p, miR-155-5p, and miR-221-3p $\left(P=4.6 \times 10^{-3}, 6.5 \times 10^{-3}\right.$, and $3.4 \times 10^{-2}$, respectively). Furthermore, a 2 miRNA-based classifier discriminated individuals with progressive disease upon TKI treatment (AUC $=0.75$, $95 \% \mathrm{Cl}, 0.64-0.85 ; P=1.3 \times 10^{-4}$ ) with better predictive value than clinicopathological risk factors commonly used. We also identified miRNAs significantly associated with progression-free survival and overall survival ( $P=6.8 \times 10^{-8}$ and $7.8 \times 10^{-7}$ for top hits, respectively), and 7 overlapped with early progressive disease. In conclusion, this is the first miRNome comprehensive study, to our knowledge, that demonstrates a predictive value of miRNAs for TKI response and provides a new set of relevant markers that can help rationalize metastatic RCC treatment.

\section{Introduction}

Renal cell carcinoma (RCC) represents around 2\%-3\% of all diagnosed cancers (1). Current first-line treatment for metastatic clear cell RCC (ccRCC) includes the tyrosine kinase inhibitors (TKI) sunitinib and pazopanib. However, about $20 \%$ of patients under this anti-VEGF-targeted therapy are refractory to the drugs (2). Thus, there is an urgent need to find biomarkers that can predict therapy outcome $(3,4)$

MicroRNAs (miRNAs) belong to a group of short noncoding RNAs that act as key regulatory molecules for various biological processes, including cellular apoptosis, proliferation, and differentiation. These molecules can differentiate ccRCC from papillary and chromophobe histologies (5) and have been associated with RCC metastasis (6-8) and aggressiveness (9-15). The Cancer Genome Atlas (TCGA) project on ccRCC showed that unsupervised analysis of miRNA expression can classify tumors into 4 distinct clusters of different survival, with miR-21 showing the strongest correlation with poor overall survival (OS) (9). Studies with a smaller number of samples have also proposed miRNA signatures as markers of aggressive ccRCC (10-15), suggesting an important role for miRNAs in prognosis. However, these studies mentioned 
Table 1. Characteristics of the patients in the discovery and validation series

\begin{tabular}{|c|c|c|c|c|}
\hline \multirow[t]{2}{*}{ Characteristic } & \multicolumn{2}{|c|}{ Discovery series $(n=74)$} & \multicolumn{2}{|c|}{ Validation series $(n=64)$} \\
\hline & $n$ & $\%$ & $n$ & $\%$ \\
\hline \multicolumn{5}{|l|}{ Age at diagnosis (y) } \\
\hline \multicolumn{5}{|l|}{ Sex } \\
\hline Male & 48 & 65 & 42 & 66 \\
\hline Female & 26 & 35 & 22 & 34 \\
\hline 0 & 42 & 57 & 18 & 28 \\
\hline 1 & 30 & 41 & 37 & 58 \\
\hline 2 & 1 & 1 & 4 & 6 \\
\hline 3 & 1 & 1 & 0 & 0 \\
\hline Missing & 0 & 0 & 5 & 8 \\
\hline 4 & 13 & 18 & 3 & 5 \\
\hline$\geq 5$ & 6 & 8 & 0 & 0 \\
\hline \multicolumn{5}{|l|}{ Treatment previous to TKI } \\
\hline Yes & 16 & 22 & 0 & 0 \\
\hline No & 58 & 78 & 64 & 100 \\
\hline \multicolumn{5}{|l|}{ TKI treatment } \\
\hline Sunitinib & 49 & 66 & 64 & 100 \\
\hline Pazopanib & 14 & 19 & 0 & 0 \\
\hline Sorafenib & 11 & 15 & 0 & 0 \\
\hline \multicolumn{5}{|l|}{ MSKCC risk group } \\
\hline Good & 13 & 18 & 36 & 56 \\
\hline Unknown & 0 & 0 & 6 & 9 \\
\hline
\end{tabular}

CR, complete response; PR, partial response; SD, stable disease; PD, progressive disease.

analyze very heterogeneous patient populations - including individuals with diverse treatments at various disease stages - and are inadequate to identify treatment response markers.

miRNAs act as regulators of hypoxia and angiogenesis (16), suggesting that they could influence the response of ccRCC to antiangiogenic drugs. This is supported by 3 exploratory studies on tumor miRNAs that, through quantitative PCR (qPCR), analyzed metastatic ccRCC cases treated with sunitinib. One study on 30 cases indicated that miR-221/222 was associated with the patients' progression-free survival (PFS) (17), another on 20 tumors proposed miR-141 as a marker for poor response to sunitinib (18), and the analysis of 6 extreme responders suggested a potential role for several miRNAs (19). However, these studies have noncoincident results and are limited by the small number of patients included and the detection of only a subset of miRNAs.

This work represents the first miRNA next-generation sequencing (NGS) study in a large cohort of ccRCC patients uniformly treated with TKIs, exploring the predictive value of these regulatory molecules. We propose TKI response markers, validate top miRNAs in an independent series, and develop combination models to accurately identify patients with a high risk of early progressive disease (PD) upon TKI treatment. 
Table 2. Top 29 miRNAs associated with PD as best objective response in ccRCC patients treated with TKIs

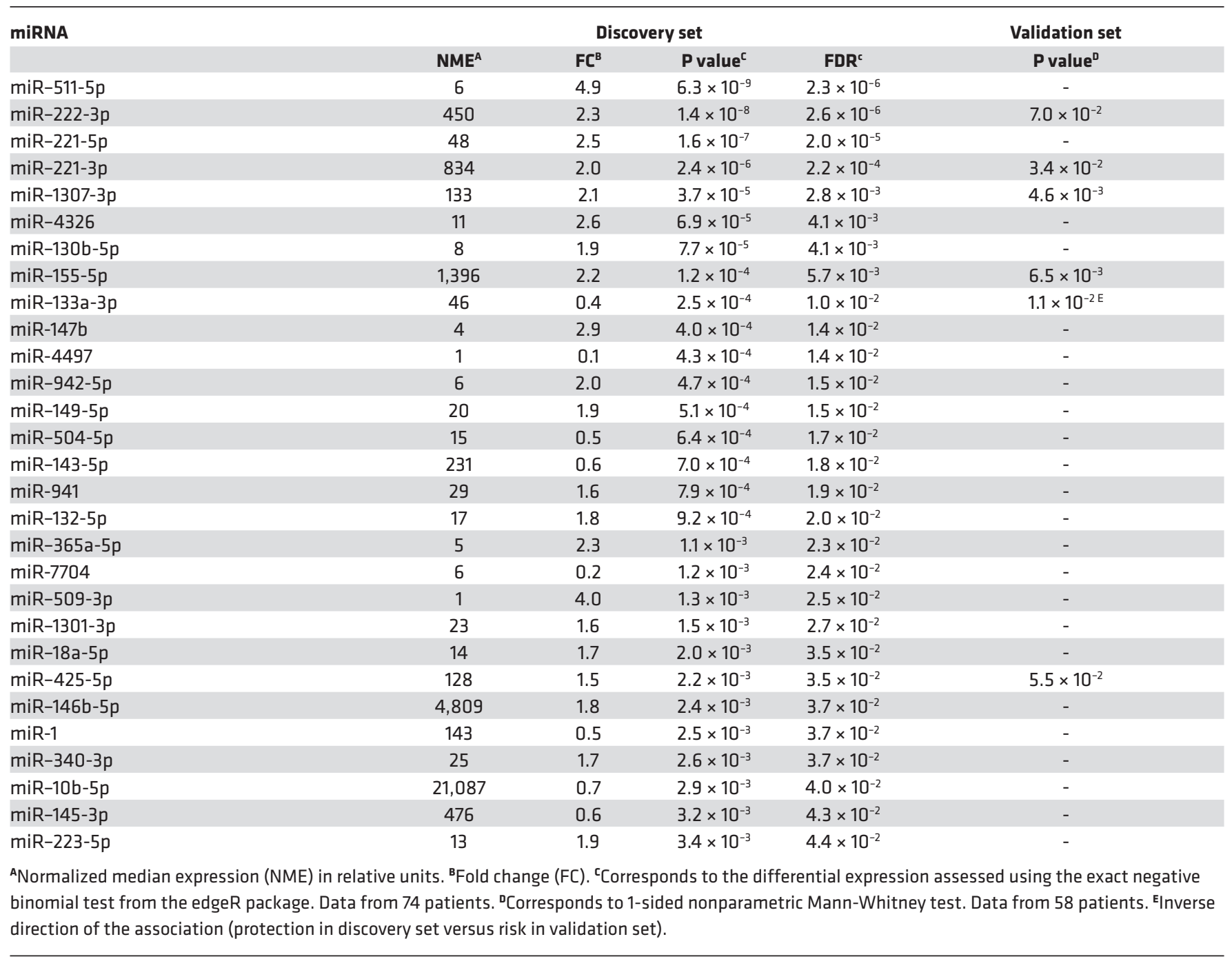

\section{Results}

miRNAs associated with TKI tumor response. Table 1 shows detailed clinicopathological characteristics of the 74 ccRCC patients treated with TKIs and with measurable disease included in the discovery series. Sixteen cases $(22 \%)$ corresponded to patients who, under TKI therapy, presented PD at first radiological assessment. The median follow-up was 49.9 months (interquartile range [IQR] $=29-77)$, and 60 patients $(81 \%)$ developed tumor progression during the follow-up period.

miRNA profiling through NGS in the discovery series identified 65 miRNAs differentially expressed in tumors progressing under TKI therapy compared with tumors showing at least stable disease $(P<$ 0.05; see Supplemental Figure 1 and Supplemental Table 1; supplemental material available online with this article; doi:10.1172/jci.insight.86051DS1). Twenty-nine miRNAs had an FDR less than 0.05, and 21 of these (72\%) were upregulated in the PD group (Table 2). Among the top differentially expressed miRNAs, 10 (34\%) had a normalized median expression higher than 100, suggesting them as easily detectable biomarkers.

miRNAs with a fold change greater than or equal to 2.0 or less than or equal to 0.5 , FDR values less than 0.01 , and a normalized median expression greater than or equal to 100 were selected for validation (i.e., miR-222-3p, miR-221-3p, miR-1307-3p, and miR-155-5p). In addition, based on literature evidence, miR-133a-3p and miR-425-5p - 2 miRNAs suggested to regulate hypoxia (20) and TKI response (17) - were also chosen for quantification in the validation series. As shown in Table 2, miR-1307-3p, 


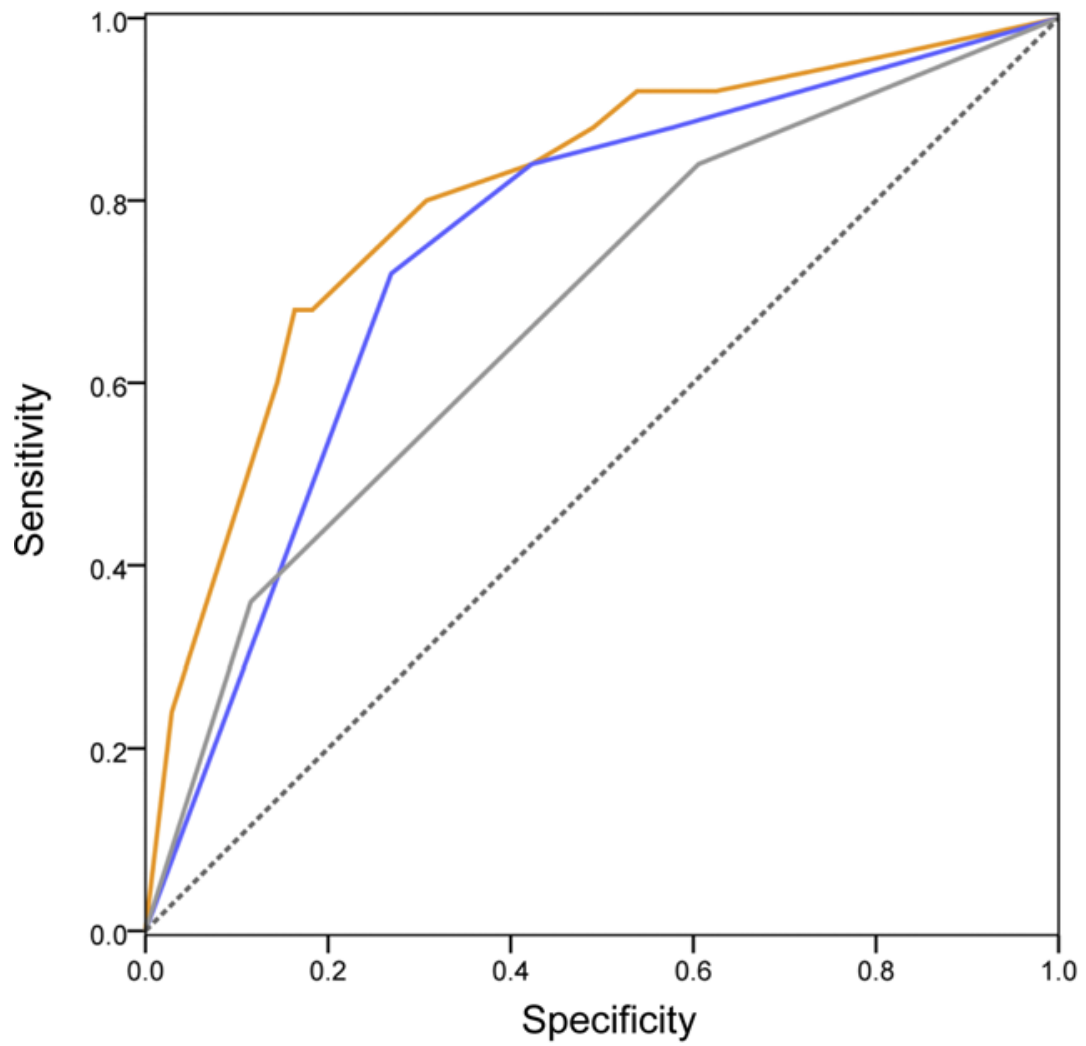

Combined miRNA and MSKCC prog. classifier
- miRNA (miR-425,miR-1307) classifier
MSKCC prognostic group
Figure 1. Receiver operating characteristic (ROC) curve analysis showing the accuracy of the miRNA-based classifier to discriminate clear cell renal cell carcinoma (ccRCC) patients with and without early progression of the disease upon tyrosine kinase inhibitor (TKI) treatment. The AUC, $95 \% \mathrm{CI}$, and $P$ value were: $\mathrm{AUC}=0.75,95 \% \mathrm{Cl}=0.64-0.85, P=1.3 \times$ $10^{-4}$ for miR-1307-3p and miR-425-5p classifier; AUC = $0.67,95 \% \mathrm{Cl}=0.56-0.80, P=5.6 \times 10^{-3}$ for the Memorial Sloan Kettering Cancer Center (MSKCC) prognostic group classifier; and AUC $=0.81,95 \% \mathrm{Cl}=0.71-0.90$, $P=2.3 \times 10^{-6}$ for the combination of miR-1307-3p, miR-425-5p, and MSKCC prognosis. Data correspond to all samples with RECIST data in the discovery plus validation series $(n=132)$.

miR-155-5p, miR-221-3p, miR-425-5p, and miR-222-3p were risk factors for PD under TKI treatment in both discovery and validation series, whereas miR-133a-3p showed opposite results among the series. The most significant $P$ values corresponded to miR-1307-3p, miR-155-5p, and miR-221-3p $\left(4.6 \times 10^{-3}, 6.5 \times 10^{-3}\right.$, and $3.4 \times 10^{-2}$, respectively; Supplemental Figure 2).

When cases were stratified according to sarcomatoid component, Memorial Sloan Kettering Cancer Center (MSKCC) prognosis, and the Stage Size Grade and Necrosis (SSIGN) score, the 5 miRNAs suggested as markers remained associated with TKI response in most of the subgroups of patients (Supplemental Table 2); changes in $P$ values may be influenced by the reduction in sample size.

Predictive model for TKI response. We generated a model predictive of TKI tumor response using a stepwise logistic regression selection method in a total cohort of 132 patients. We considered the 5 miRNAs that the validation set suggested as markers of poor TKI response and included in the analysis relevant clinical characteristics (MSKCC prognostic group, age, sex, and time from nephrectomy to TKI). The selected miRNA predictive model included miR-1307-3p and miR-425-5p, which were associated with TKI response both in univariate (odds ratio $[\mathrm{OR}]=4.3,95 \% \mathrm{CI}, 1.6-11.7, P=4.0 \times 10^{-3}$, and $\mathrm{OR}=7.2$, 95\% CI, 2.3-22.3; $P=7.0 \times 10^{-4}$, respectively), and multivariable analysis adjusting for MSKCC prognostic group $\left(\mathrm{OR}=4.9,95 \% \mathrm{CI}, 1.7-13.9 ; P=2.9 \times 10^{-3}\right.$, and $\mathrm{OR}=6.5,95 \% \mathrm{CI}, 2.0-20.9 ; P=1.6 \times$ $10^{-3}$, respectively). In the final predictive model, an increased expression of miR-1307-3p and miR-425-5p was associated with an increased risk of PD upon TKI treatment, with risk score $=(1.11 \times$ miR-1307-3p $)$ $+(1.48 \times$ miR-425-5p) $+(1.06 \times$ MSKCC group $)-4.09$, where low expression status equals 0 and high expression equals 1 . We assessed the accuracy of the miRNA-based classifier with receiver operating characteristic (ROC) analysis (Figure 1). The miRNA-based classifier showed higher accuracy than any clinical factor tested, and as shown above, adding the MSKCC prognostic group to the final model increased the predictive value (the AUC for the miRNA-based model was 0.75 and augmented to 0.81 when including the prognostic group).

PFS and OS upon TKI treatment. We identified miRNAs associated with the PFS and OS of the TKI-treated patients using the miRNome data from the discovery series. As shown in Figure 2, no miRNA was exclusively associated with PFS, while 24 were exclusively associated with OS, 5 were associated simultaneously with OS and PFS, 8 were associated with OS and PD as best tumor response, and $7 \mathrm{miR}$ NAs were simultaneously associated with worse OS, PFS, and PD as best TKI response. This latter group, which contained the vascular remodeling miR-221/222 gene cluster, defined a signature of miRNAs associated with TKI-resistant tumors with a poor prognosis. For example, as shown in Table 3, miR-221-3p and miR-222-3p were associated with PFS with $P$ values of $6.8 \times 10^{-8}$ and $2.3 \times 10^{-6}$ and were associated 


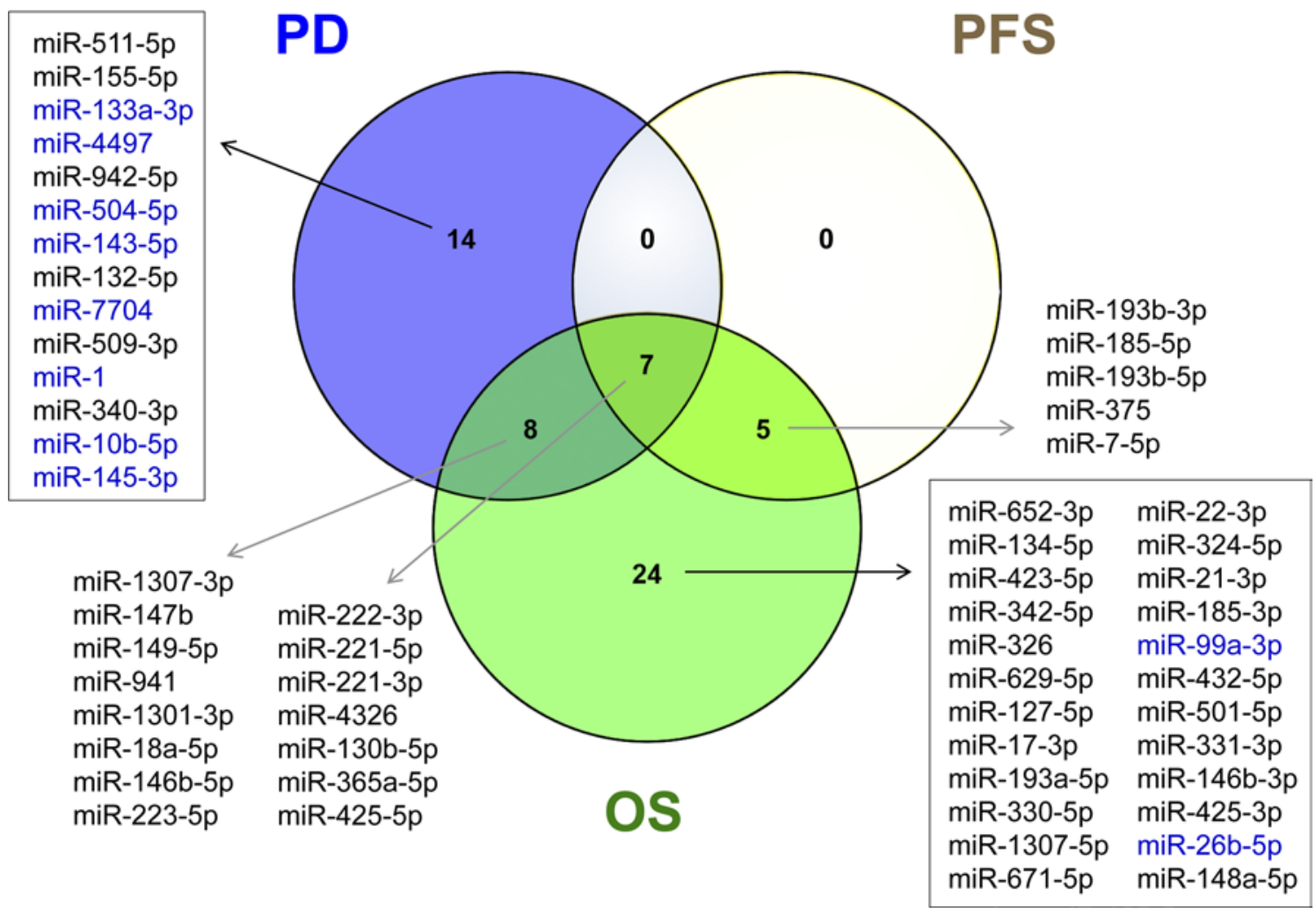

Figure 2. Venn diagram of miRNAs associated with progressive disease (PD) as best response, progression-free survival (PFS), and overall survival (OS) using FDR $<\mathbf{0 . 0 5}$ as threshold. miRNAs downregulated in tumors from patients with poor outcome (PD as best response, short PFS, or OS) are shown in blue; the rest of miRNAs are overexpressed in patients with poor outcome. Data corresponds to the discovery series $(n=74)$. Venn diagrams were constructed using the tool http://bioinfogp.cnb.csic.es/tools/venny/index.html.

with OS with $P$ values of $8.9 \times 10^{-6}$ and $7.8 \times 10^{-7}$, respectively; they were overexpressed in the tumors of patients with shorter survival times.

An analysis including MSKCC prognostic classification, age, and time from nephrectomy to TKI as covariates in the analysis did not substantially change the associations of the top miRNAs with PFS and OS (Table 3 and Supplemental Tables 3 and 4). No association between sex, type of TKI, treatment previous to the TKIs, sarcomatoid component, and SSIGN score with PFS and OS was found, and inclusion of these variables in the analysis did not substantially change the results obtained.

Upon analysis of potential miRNA deregulated pathways, DNA replication was predicted to be the most significantly altered pathway by the miRNAs associated with PFS (FDR $=2 \times 10^{-19}$ ), with pathways involved in diverse types of cancers having FDRs ranging from $7 \times 10^{-15}$ to $7 \times 10^{-11}$ (Supplemental Table 5). For OS, DNA replication (FDR $\left.=8 \times 10^{-18}\right)$, p53 signaling $\left(4 \times 10^{-13}\right)$, and cancer pathways (FDR ranging from $3 \times 10^{-13}$ to $8 \times 10^{-13}$ ) were among the most deregulated ones.

\section{Discussion}

The repertoire of drugs approved for metastatic RCC is rapidly growing; however, there is a lack of biomarkers that can guide therapy with these drugs. The aim of this study was to explore whether miRNAs could be used as TKI predictive markers to personalize ccRCC treatment. We generated the first miRNome data to our knowledge on a large ccRCC series uniformly treated and identified, and we validated miRNAs significantly associated with PD under TKI. Furthermore, we established a miRNA-based classifier, with a better predictive value than clinicopathological risk factors commonly used, able to discriminate TKI refractory patients. In addition, miRNAs associated with TKI resistance and shorter PFS and OS were also identified.

Several reports have identified miRNAs associated with ccRCC histology, metastasis, and prognosis (5-15). However, few studies with limited number of samples have investigated miRNAs in relation with TKI predictive response in ccRCC, analyzing only subsets of miRNAs with techniques that have the risk 
Table 3. Top miRNAs associated with PFS and OS in ccRCC patients treated with TKIs

\begin{tabular}{|c|c|c|c|c|c|c|c|c|c|c|c|}
\hline \multirow[t]{2}{*}{ miRNA } & \multirow[t]{2}{*}{ NME $^{A}$} & \multicolumn{5}{|c|}{ PFS $^{B}$} & \multicolumn{5}{|c|}{ OS $^{B}$} \\
\hline & & Rank & $\mathrm{HR}(95 \% \mathrm{Cl})$ & FDR univ. ${ }^{\mathrm{C}}$ & $P$ univ. & $P$ multiv. ${ }^{D}$ & Rank & HR (95\% CI) & FDR univ. & P univ. & $P$ multiv. \\
\hline miR-221-3p & 834 & 1 & $2.25(1.67-3.02)$ & $2.5 \times 10^{-5}$ & $6.8 \times 10^{-8}$ & $3.8 \times 10^{-6}$ & 7 & $1.71(1.35-2.16)$ & $4.7 \times 10^{-4}$ & $8.9 \times 10^{-6}$ & $1.1 \times 10^{-3}$ \\
\hline miR-221-5p & 48 & 3 & $1.60(1.30-1.98)$ & $1.3 \times 10^{-3}$ & $1.0 \times 10^{-5}$ & $9.8 \times 10^{-5}$ & 15 & $1.23(1.10-1.37)$ & $8.7 \times 10^{-3}$ & $3.7 \times 10^{-4}$ & $6.0 \times 10^{-3}$ \\
\hline$m i R-425-5 p$ & 128 & 4 & $1.72(1.32-2.25)$ & $5.7 \times 10^{-3}$ & $6.2 \times 10^{-5}$ & $1.1 \times 10^{-3}$ & 11 & $1.63(1.27-2.09)$ & $4.5 \times 10^{-3}$ & $1.4 \times 10^{-4}$ & $1.2 \times 10^{-3}$ \\
\hline miR-4326 & 11 & 5 & $1.37(1.16-1.62)$ & $1.1 \times 10^{-2}$ & $1.5 \times 10^{-4}$ & $1.7 \times 10^{-3}$ & 9 & $1.37(1.18-1.60)$ & $2.3 \times 10^{-3}$ & $5.6 \times 10^{-5}$ & $3.6 \times 10^{-3}$ \\
\hline miR-375 & 3 & 8 & $1.06(1.03-1.10)$ & $2.0 \times 10^{-2}$ & $4.4 \times 10^{-4}$ & $1.3 \times 10^{-2}$ & 2 & $1.10(1.06-1.14)$ & $1.5 \times 10^{-4}$ & $9.7 \times 10^{-7}$ & $9.6 \times 10^{-4}$ \\
\hline miR-130b-5p & 8 & 9 & $1.60(1.23-2.09)$ & $2.0 \times 10^{-2}$ & $5.2 \times 10^{-4}$ & $2.4 \times 10^{-3}$ & 42 & $1.43(1.11-1.84)$ & $4.5 \times 10^{-2}$ & $5.3 \times 10^{-3}$ & $6.5 \times 10^{-2}$ \\
\hline miR-185-5p & 338 & 10 & $1.65(1.24-2.19)$ & $2.0 \times 10^{-2}$ & $5.9 \times 10^{-4}$ & $1.1 \times 10^{-2}$ & 4 & $2.13(1.54-2.94)$ & $4.3 \times 10^{-4}$ & $4.7 \times 10^{-6}$ & $8.2 \times 10^{-4}$ \\
\hline miR-193b-5p & 5 & 11 & $1.12(1.05-1.20)$ & $2.0 \times 10^{-2}$ & $6.1 \times 10^{-4}$ & $9.4 \times 10^{-3}$ & 3 & $1.21(1.12-1.30)$ & $1.5 \times 10^{-4}$ & $1.2 \times 10^{-6}$ & $1.1 \times 10^{-4}$ \\
\hline
\end{tabular}

ANormalized median expression (NME) in relative units. ${ }^{B} \mathrm{PFS}$ and $0 \mathrm{~S}$ were analyzed through Cox-regression analysis in 74 patients.

'Univariate analysis (univ.). ${ }^{\circ}$ Multivariable analysis (multiv.) includes as covariables MSKCC prognosis, age, and time from nephrectomy to TKI treatment.

for biases. In this study, we applied NGS, an unbiased method that accurately quantifies whole miRNome expression, to a large series of uniformly treated ccRCC patients. We identified 29 miRNAs predictive of TKI response and selected for validation potentially actionable miRNAs with strong effects - since only abundant miRNAs within a cell may mediate significant target suppression (21) — and miRNAs previously suggested to influence TKI response. Among the 6 miRNAs selected for validation, we obtained confirmatory results for miR-1307, which had not been previously related with RCC or TKI response, and for miR155, miR-221, miR-425, and miR-222, for which preliminary evidence suggesting an influence on sunitinib outcome exists $(17,18)$. Concerning miR-133a, which is suggested to regulate hypoxia $(20)$, we found contradictory results in the discovery and validation set. Interestingly, a previous study with $6 \mathrm{ccRCC}$ patients with extreme responses to sunitinib (19) suggested downregulation of miR-133a in poor response patients, in line with our discovery series. However, the independent set used for confirmation, the same used here for validation, and a sunitinib-resistant cell line suggested miR-133a overexpression in the tumors of TKI refractory patients (19). Variability among the series may be related to these differences. For example, in the discovery series, $27 \%$ of patients were in the poor MSKCC prognostic group and $22 \%$ received treatments previous to the TKIs; in the validation series, only 1 patient had poor prognosis and all received sunitinib as first-line treatment. At any rate, further work is needed to clarify this. It is also important to highlight that, among the 29 miRNAs we found associated with TKI refractoriness, many have previously been shown to play a role in angiogenesis - e.g., $\operatorname{miR}-221 / 222(22,23), \operatorname{miR}-155-5 p(24,25)$, miR-143-5p (26), miR-132-5p (27), miR-1 (28), miR-10b-5p (29), and miR-145-3p (26) — and several have been associated with RCC metastasis - e.g., miR-221/222 (7), miR-130b (30), miR-1 (31), and miR-10b-5p (30). Many of these miRNAs, although not selected for validation either due to low expression or weaker association values, may also constitute additional markers of TKI response.

Furthermore, using the full set of patients in the discovery and validation cohorts, we established a combination model that included 2 miRNAs (miR-1307-3p and miR-425-5p) and the MSKCC prognostic score to discriminate TKI refractory patients (AUC $=0.81 ; P=2.3 \times 10^{-6}$ ). Among these 2 miRNAs, miRNA-425 has been associated with tumor stage in gastric (32) and lung cancer (33), and in RCC, it has been suggested as being a potential ccRCC biomarker (34) associated with poor prognosis for chromophobe RCC (35) and decreased PFS during sunitinib treatment (17). miR-1307 plays a role in chemoresistance in ovarian cancer (36), and it has been suggested to contribute to colorectal carcinogenesis (37). Its role in $\mathrm{RCC}$ is less clear, since its association with this tumor has not been described before. Therefore, further studies are required to elucidate the biological role of this miRNA. 
Concerning ccRCC prognosis, several of the miRNAs associated with OS in this study are in line with findings from other groups (Supplemental Table 4). For example, overexpression of miR-21 has been associated with poor OS in TCGA (9) and in other studies $(10,14)$. In this study, miR-21 was not among the top-ranking miRNA associated with OS, but it was significantly associated with shorter OS $(P=3.4 \times$ $10^{-3}$ ). From our OS top-associated miRNAs (e.g., miR-222-3p, miR-375, miR-193b-5p, miR-185-5p, and miR-193b-3p), miR-222 has already been shown to be associated with poor OS in RCC (15). The other miRNAs, however, represent prognostic markers not described before. With respect to the underlying pathways altered, the fact that the miRNAs associated with OS are involved in DNA replication and cell cycle makes their role in RCC progression conceivable.

In the absence of a placebo-treated group of patients, we cannot define whether the miRNAs associated here with PFS and OS have a predictive and/or a prognostic value. Nevertheless, 7 miRNAs (miR-222-3p, miR-221-5p, miR-221-3p, miR-4326, miR-130b-5p, miR-365a-5p, and miR-425-5p) defined a group of patients not only associated with short PFS and OS, but also with poor TKI response. In these patients, the shorter PFS and OS are probably, at least in part, the consequence of poor efficacy of the anti-VEGFR-TKI. The miR-221/222 gene cluster, which has already been associated with poor prognosis in ccRCC $(15,38)$, showed the strongest association. Its dual effect, in poor prognosis and TKI refractoriness, is supported by the key role of miR-221/222 in cell proliferation, invasion, metastasis (39), and angiogenesis (22, 23). VEGFR2 is a direct target of miR-221/222 (17), suggesting that depletion of this TKI target would render these drugs less effective. This subset of patients might therefore be more effectively treated with alternative drugs (e.g., immune checkpoint inhibitors).

With respect to the possible limitations imposed by ccRCC intratumoral heterogeneity (40), if TKI response is driven by specific clonal populations, these might not be sufficiently represented in single tumor blocks (41). However, the miRNAs here proposed as markers were validated internally and also by external studies that did not use multiregion sampling. Currently, it is unknown if the expression of these miRNAs is subjected to intratumor variability (e.g., if the deregulation of the miRNAs is an early RCC event, similar to the VHL mutation in ref. 40, no variability is expected). And if there is variability, it is unknown how it would influence clinical outcome. The use of miRNAs as predictive markers in ccRCC is further supported by the fact that an mRNA gene-expression signature affected by intratumor heterogeneity has been shown to outperform genetic biomarkers for the prediction of ccRCC cancer-specific survival and to add prognostic information to tumor stage and SSIGN prognostic model $(42,43)$.

In conclusion, this first miRNome deep-sequencing study on a well-characterized series of ccRCC patients uniformly treated reveals miRNAs as predictive markers for TKI response. The results of this study argue for a prospective validation of miRNA expression in patients undergoing TKI therapy and suggest that metastatic ccRCC therapy could be further personalized.

\section{Methods}

Patients. In the tumor bank of the University Hospitals Leuven, we searched for metastatic ccRCC patients treated with sunitinib, sorafenib, or pazopanib as first-line anti-VEGFR-TKIs with available archived formalin-fixed paraffin embedded (FFPE) primary tumors and nephrectomy as first therapeutic intervention. Previous immunotherapy or chemotherapy was allowed, but previous exposure to other targeted therapies was an exclusion criterion. miRNA NGS was performed in 81 cases, but 5 were subsequently excluded because no CT scan evaluating anti-VEGFR-TKI response was available (they all had less than 1 month TKI treatment), 1 case was a misclassified papillary RCC, and 1 patient developed a secondary tumor potentially affecting TKI response. Therefore, 74 ccRCCs were included in the analysis (Table 1).

Validation of results was performed using a series of 64 FFPE primary tumors with clear cell histology and tumor RNA material available, derived from a study of 101 metastatic patients treated with first-line sunitinib $(44,45)$. The clinical outcome of these patients, objective response rate, PFS, and OS has been reported previously $(44,45)$. The main characteristics of these patients are presented in Table 1.

$R N A$ isolation. H\&E-stained sections of the tumor samples from discovery and validation series were examined by a pathologist (M. Morente) to confirm the diagnosis and to estimate tumor content. In the discovery series, tumor content was above $90 \%$ in 71 cases and was $70 \%$ in 3 cases. Total RNA was isolated with the Recover All Total Nucleic Acid Isolation kit (Ambion) using 4-6 whole 10- $\mu \mathrm{m}$ sections of the tumor samples. In the validation series, total RNA was isolated using the RNeasy FFPE kit (QIAGEN) using selected areas representative of each tumor (45). RNA quantity and quality were 
assessed by NanoDrop Spectrophotometer (Nanodrop Technologies).

NGS. Purified total RNA $(0.5 \mu \mathrm{g})$ was used to produce cDNA-amplified libraries using the NEBNext Multiplex Small RNA Library Prep for Illumina (New England Biolabs). Libraries were sequenced for 50 bases in a single-read format (Genome Analyzer IIx, Illumina). Reads were quality checked with FastQC (http://www.bioinformatics.babraham.ac.uk/projects/fastqc). Adapter sequences were removed with cutadapt v1.2.1 (http://journal.embnet.org/index.php/embnetjournal/article/view/200), and only those reads longer than $15 \mathrm{bp}$ and shorter than $35 \mathrm{bp}$ were kept for further analysis. Reads were aligned to the human genome (GRCh37/hg19) with Bowtie 0.12.7 (46) and Samtools 0.1.18 (47), allowing no mismatches and a maximum of 1 alignment per read. Raw counts for miRNAs were obtained with HTSeq v0.5.3p9 (48), using the miRBase v20 annotation (49) for hg19. On average, 13 million reads were obtained per sample. The data derived from this work have been deposited in the NCBI's Gene Expression Omnibus (GEO; GSE74174).

$q P C R$. qPCR analysis was performed in the validation series using the Universal miRCURY LNA microRNA PCR System (Exiqon), following the manufacturer's instructions (see Supplementary Materials and Methods for further details).

Statistics. Statistics was performed in R version 3.1.2. Raw counts were obtained for 2576 miRNAs and normalized with DESeq Bioconductor package (50). Those miRNAs that had a normalized count value below 15 in greater than or equal to $95 \%$ of the samples were considered to be of low expression and were filtered out, reducing the initial number of miRNAs to 374. Differential expression in the discovery series between tumors with PD as best response to TKI treatment (according to Response Evaluation Criteria In Solid Tumors [RECIST] criteria) and those with other responses were assessed using the exact negative binomial test from the edgeR package (50). $P$ values were corrected for multiple hypotheses testing using the Benjamini and Hochberg FDR adjustment (51).

Models correlating TKI response and miRNA expression were constructed using stepwise conditional logistic regression analysis with the total population. miRNAs included in the analysis were those with the same effect (protective/risk) in discovery and validation series. To combine the data, the expression of the miRNAs was expressed as a dichotomous variable using the median miRNA expression as a threshold in each series. To avoid a possible series effect, a binary variable that identified each series was included in the analysis. The discrimination power of the model was evaluated by computing ROC curves and AUC.

We assessed miRNAs associated with PFS and disease-specific OS using NGS data through Cox-regression modeling ( $\mathrm{R}$ package survival). We defined PFS as the time between the first day of treatment with TKI and the date of radiological or clinical PD or clear clinical evidence of PD. We defined disease-specific OS as the time between the first day of treatment with TKI and patient death due to disease. Patients who were lost to follow-up or had not progressed at the time of the study were treated as censored events in the PFS analysis. For OS analysis, living patients or those lost to follow-up were censored. To have comparable miRNA effect magnitudes in the survival analyses, the expression of each miRNA was divided by its median expression value. Both univariate and multivariable PFS and OS analysis were performed; multivariable analysis included MSKCC prognostic classification, age, and time from nephrectomy to TKI as covariates.

To identify the target pathways of the miRNAs associated with TKI refractoriness, PFS, and OS, we used the DIANA miRPath software, using experimentally validated miRNA interactions derived from DIANA-TarBase v6.0 predictions.

For differential expression and survival analysis, $P$ values were corrected using FDR adjustment (51) and an FDR less than 0.05 was considered significant. For ROC curve analysis, a $P$ value less than 0.05 was considered significant. In the differential expression analysis for the validation, we applied a 1-sided Mann-Whitney test, based on the assumption that miRNAs had to follow the same direction as in the discovery series.

Study approval. The IRBs of all involved institutions approved the study. Written informed consent was obtained from all patients alive. In the case patients had died when the study started, a general positive advice for the utilization of remaining tissue was foreseen by the institutional board.

\section{Author contributions}

JGD, BB, and CRA designed the research study. LIP, OG, DGP, MAR, LJLG, and MM conducted experiments. JGD, BB, PS, AW, OB, EE, DEC, AGDA, MAC, SH, and JAA acquired the data. LIP, OG, MR, and CRA performed the analysis and interpretation of data. JGD, BB, and CRA supervised the study, and all authors contributed to the writing, review, and revision of the manuscript. 


\section{Acknowledgments}

This study was supported by the Fundación Mutua Madrileña, by an unrestricted educational grant from Pfizer (WI194736/SUT-IIG-25), by Fondo de Investigaciones Sanitarias Project PI13/0622, and by the Spanish Ministry of Economy and Competitiveness (SAF2015-70820-ERC). María Apellániz-Ruiz is a predoctoral fellow of "la Caixa"/CNIO international $\mathrm{PhD}$ programme. The authors appreciate the collaboration of the participating patients and their families. We thank M. Victoria Bolós for her support in the project.

Address correspondence to: Cristina Rodriguez-Antona, Hereditary Endocrine Cancer Group, Human Cancer Genetics Programme, Spanish National Cancer Research Centre, Melchor Fernández Almagro 3; 28029 Madrid, Spain. Phone: 34.91.732.8000; Fax: 34.91.224.6911; E-mail: crodriguez@cnio.es.

1. Gupta K, Miller JD, Li JZ, Russell MW, Charbonneau C. Epidemiologic and socioeconomic burden of metastatic renal cell carcinoma (mRCC): a literature review. Cancer Treat Rev. 2008;34(3):193-205.

2. Motzer RJ, et al. Sunitinib versus interferon alfa in metastatic renal-cell carcinoma. N Engl J Med. 2007;356(2):115-124.

3. Rodríguez-Antona C, García-Donas J. Constitutional genetic variants as predictors of antiangiogenic therapy outcome in renal cell carcinoma. Pharmacogenomics. 2012;13(14):1621-1633.

4. Garcia-Donas J, Rodriguez-Moreno JF, Romero-Laorden N, Rodriguez-Antona C. Renal carcinoma pharmacogenomics and predictors of response: Steps toward treatment individualization. Urol Oncol. 2015;33(4):179-186.

5. Spector Y, et al. Development and validation of a microRNA-based diagnostic assay for classification of renal cell carcinomas. Mol Oncol. 2013;7(3):732-738.

6. Wotschofsky Z, et al. Diagnostic and prognostic potential of differentially expressed miRNAs between metastatic and non-metastatic renal cell carcinoma at the time of nephrectomy. Clin Chim Acta. 2013;416:5-10.

7. Heinzelmann J, et al. Specific miRNA signatures are associated with metastasis and poor prognosis in clear cell renal cell carcinoma. World J Urol. 2011;29(3):367-373.

8. Heinzelmann J, et al. MicroRNAs with prognostic potential for metastasis in clear cell renal cell carcinoma: a comparison of primary tumors and distant metastases. Ann Surg Oncol. 2014;21(3):1046-1054.

9. Cancer Genome Atlas Research Network. Comprehensive molecular characterization of clear cell renal cell carcinoma. Nature. 2013;499(7456):43-49.

10. Ge YZ, et al. A tumor-specific microRNA signature predicts survival in clear cell renal cell carcinoma. J Cancer Res Clin Oncol. 2015;141(7):1291-1299.

11. Fritz HK, Lindgren D, Ljungberg B, Axelson H, Dahlbäck B. The miR(21/10b) ratio as a prognostic marker in clear cell renal cell carcinoma. Eur J Cancer. 2014;50(10):1758-1765.

12. Mangolini A, et al. Differential expression of microRNA501-5p affects the aggressiveness of clear cell renal carcinoma. FEBS Open Bio. 2014;4:952-965.

13. Fu Q, et al. Tumor miR-125b predicts recurrence and survival of patients with clear-cell renal cell carcinoma after surgical resection. Cancer Sci. 2014;105(11):1427-1434.

14. Vergho D, et al. Combination of expression levels of miR-21 and miR-126 is associated with cancer-specific survival in clear-cell renal cell carcinoma. BMC Cancer. 2014;14:25.

15. Teixeira AL, et al. Higher circulating expression levels of miR-221 associated with poor overall survival in renal cell carcinoma patients. Tumour Biol. 2014;35(5):4057-4066.

16. Suárez Y, Sessa WC. MicroRNAs as novel regulators of angiogenesis. Circ Res. 2009;104(4):442-454.

17. Khella HW, et al. miR-221/222 are involved in response to sunitinib treatment in metastatic renal cell carcinoma. Mol Ther. 2015;23(11):1748-1758.

18. Berkers J, et al. A possible role for microRNA-141 down-regulation in sunitinib resistant metastatic clear cell renal cell carcinoma through induction of epithelial-to-mesenchymal transition and hypoxia resistance. J Urol. 2013;189(5):1930-1938.

19. Prior C, et al. Identification of tissue microRNAs predictive of sunitinib activity in patients with metastatic renal cell carcinoma PLoS ONE. 2014;9(1):e86263.

20. Li AY, Yang Q, Yang K. miR-133a mediates the hypoxia-induced apoptosis by inhibiting TAGLN2 expression in cardiac myocytes. Mol Cell Biochem. 2015;400(1-2):173-181.

21. Mullokandov G, et al. High-throughput assessment of microRNA activity and function using microRNA sensor and decoy libraries. Nat Methods. 2012;9(8):840-846.

22. Urbich C, Kuehbacher A, Dimmeler S. Role of microRNAs in vascular diseases, inflammation, and angiogenesis. Cardiovasc Res. 2008;79(4):581-588.

23. Nicoli S, Knyphausen CP, Zhu LJ, Lakshmanan A, Lawson ND. miR-221 is required for endothelial tip cell behaviors during vascular development. Dev Cell. 2012;22(2):418-429.

24. Pankratz F, et al. MicroRNA-155 exerts cell-specific antiangiogenic but proarteriogenic effects during adaptive neovascularization. Circulation. 2015;131(18):1575-1589.

25. Donnem T, et al. MicroRNA signatures in tumor tissue related to angiogenesis in non-small cell lung cancer. PLoS ONE. 2012;7(1):e29671.

26. Climent M, Quintavalle M, Miragoli M, Chen J, Condorelli G, Elia L. TGF $\beta$ Triggers miR-143/145 transfer from smooth muscle cells to endothelial cells, thereby modulating vessel stabilization. Circ Res. 2015;116(11):1753-1764.

27. Anand S, et al. MicroRNA-132-mediated loss of p120RasGAP activates the endothelium to facilitate pathological angiogenesis Nat Med. 2010;16(8):909-914. 
28. Lin CY, et al. MiR-1 and miR-206 target different genes to have opposing roles during angiogenesis in zebrafish embryos. Nat Commun. 2013;4:2829.

29. Plummer PN, et al. MicroRNAs regulate tumor angiogenesis modulated by endothelial progenitor cells. Cancer Res. 2013;73(1):341-352.

30. Wu X, et al. Identification of a 4-microRNA signature for clear cell renal cell carcinoma metastasis and prognosis. PLoS ONE 2012;7(5):e35661.

31. Xiao H, et al. MiR-1 downregulation correlates with poor survival in clear cell renal cell carcinoma where it interferes with cell cycle regulation and metastasis. Oncotarget. 2015;6(15):13201-13215.

32. Zhang Z, et al. microRNA-425-5p is upregulated in human gastric cancer and contributes to invasion and metastasis in vitro and in vivo. Exp Ther Med. 2015;9(5):1617-1622.

33. Wang J, et al. Characterization of microRNA transcriptome in tumor, adjacent, and normal tissues of lung squamous cell carcinoma. J Thorac Cardiovasc Surg. 2015;149(5):1404-1414.e4.

34. Hao JF, et al. Identification of potential biomarkers for clear cell renal cell carcinoma based on microRNA-mRNA pathway relationships. J Cancer Res Ther. 2014;10 Suppl:C167-C177.

35. Ge YZ, et al. MicroRNA expression profiles predict clinical phenotypes and prognosis in chromophobe renal cell carcinoma. Sci Rep. 2015;5:10328.

36. Zhou Y, Wang M, Wu J, Jie Z, Chang S, Shuang T. The clinicopathological significance of miR-1307 in chemotherapy resistant epithelial ovarian cancer. J Ovarian Res. 2015;8:23.

37. Tang R, et al. The polymorphic terminal-loop of pre-miR-1307 binding with MBNL1 contributes to colorectal carcinogenesis via interference with Dicer1 recruitment. Carcinogenesis. 2015;36(8):867-875.

38. Teixeira AL, et al. Combined influence of $\mathrm{EGF}+61 \mathrm{G}>\mathrm{A}$ and $\mathrm{TGFB}+869 \mathrm{~T}>\mathrm{C}$ functional polymorphisms in renal cell car cinoma progression and overall survival: the link to plasma circulating MiR-7 and MiR-221/222 expression. PLoS ONE. 2014;10(4):e0103258.

39. Garofalo M, Quintavalle C, Romano G, Croce CM, Condorelli G. miR221/222 in cancer: their role in tumor progression and response to therapy. Curr Mol Med. 2012;12(1):27-33.

40. Gerlinger M, et al. Genomic architecture and evolution of clear cell renal cell carcinomas defined by multiregion sequencing. Nat Genet. 2014;46(3):225-233.

41. Sankin A, et al. The impact of genetic heterogeneity on biomarker development in kidney cancer assessed by multiregional sampling. Cancer Med. 2014;3(6):1485-1492.

42. Brannon AR, et al. Molecular stratification of clear cell renal cell carcinoma by consensus clustering reveals distinct subtypes and survival patterns. Genes Cancer. 2010;1(2):152-163.

43. Gulati S, et al. Systematic evaluation of the prognostic impact and intratumour heterogeneity of clear cell renal cell carcinoma biomarkers. Eur Urol. 2014;66(5):936-948.

44. Garcia-Donas J, et al. Single nucleotide polymorphism associations with response and toxic effects in patients with advanced renal-cell carcinoma treated with first-line sunitinib: a multicentre, observational, prospective study. Lancet Oncol. 2011;12(12):1143-1150.

45. Garcia-Donas J, et al. Prospective study assessing hypoxia-related proteins as markers for the outcome of treatment with sunitinib in advanced clear-cell renal cell carcinoma. Ann Oncol. 2013;24(9):2409-2414.

46. Langmead B, Trapnell C, Pop M, Salzberg SL. Ultrafast and memory-efficient alignment of short DNA sequences to the human genome. Genome Biol. 2009;10(3):R25.

47. Li H, et al. The Sequence Alignment/Map format and SAMtools. Bioinformatics. 2009;25(16):2078-2079.

48. Anders S, Pyl PT, Huber W. HTSeq--a Python framework to work with high-throughput sequencing data. Bioinformatics. 2015;31(2):166-169.

49. Kozomara A, Griffiths-Jones S. miRBase: annotating high confidence microRNAs using deep sequencing data. Nucleic Acids Res. 2014;42(Database issue):D68-D73.

50. Anders S, et al. Count-based differential expression analysis of RNA sequencing data using R and Bioconductor. Nat Protoc. 2013;8(9):1765-1786.

51. Benjamini Y, Hochberg Y. Controlling the false discovery rate: a practical and powerful approach to multiple testing. JR Stat Soc B. 1995;57:289-300. 